\title{
Effects of potato-derived protease inhibitors on perianal dermatitis after colon resection for long-segment Hirschsprung's disease
}

\author{
Steffen Berger, Janine Rufener, Peter Klimek, Zacharias Zachariou, Colette Boillat \\ Bern, Switzerland
}

Background: After resection of long-segment Hirschsprung's disease, severe perianal dermatitis (SPAD) may occur because of high stool frequency and elevated concentrations of fecal pancreatic proteases. We investigated prospectively the effect of potato-derived protease inhibitors (PPI) on skin conditions in children with postoperative SPAD.

Methods: Four children (aged 12 to 24 months) with therapy-resistant SPAD after transanal endorectal pull-through for long-segment Hirschsprung's disease received topical PPI (1\% in $20 \%$ zinc ointment) with each diaper-change. Parents noted down a subjective dermatitis score daily. Photo documentation and outpatient visits were made to assess the treatment results.

Results: No adverse effects were observed after treatment with PPI. A remarkable improvement of the dermatitis in 3 of the 4 patients as well as improvements in pain and sleep disorders were observed during the PPI-treatment course.

Conclusion: The results of the study suggest that PPI may reduce otherwise intractable protease-induced skin irritation in infants.

World J Pediatr 2012;8(2):173-176

Key words: Hirschsprung's disease;

perianal dermatitis;

protease inhibitor;

transanal endorectal pull-through

Author Affiliations: Department of Pediatric Surgery, Children's University Hospital, Inselspital, University of Bern, Switzerland (Berger S, Rufener J, Klimek P, Zachariou Z, Boillat C)

Corresponding Author: S Berger, MD, Department of Pediatric Surgery, Children's University Hospital, Inselspital, University of Bern, CH-3010 Bern, Switzerland (Tel: 0041-31-632-9274; Fax: 0041-31-632-9292; Email: steffen.berger@insel.ch)

doi: 10.1007/s 12519-012-0356-2

(C)Children's Hospital, Zhejiang University School of Medicine, China and Springer-Verlag Berlin Heidelberg 2012. All rights reserved.

World J Pediatr, Vol 8 No 2 · May 15, 2012 · www.wjpch.com

\section{Introduction}

T $T$ ith long-segment Hirschsprung's disease, perianal skin excoriation is a common problem with any operative procedures applied $^{[1-5]}$ including transanal endorectal pull-through (TERPT). ${ }^{[6,7]}$ The temporarily increased stool frequency during the early postoperative period ${ }^{[8,9]}$ is probably due to intraoperative internal sphincter dilation, ${ }^{[10]}$ which results in a condition termed pseudo-incontinence.

Feces contain proteases such as trypsin, alphachymotrypsin and elastase which are secreted by the pancreas, the small intestinal brush-border and the stomach. ${ }^{[11]}$ The activity of these proteases is normally neutralized during the transit along the small intestine and colon. ${ }^{[12,13]}$ The proteolytic activity in feces is several times higher in newborns and infants than in adults. ${ }^{[14]}$ Proteolytic activity is also increased with a reduced intestinal passage time (e.g., after colectomy), or with diarrhea when small bowel content reaches the perianal skin. ${ }^{[14]}$

In children with long-segment Hirschsprung's disease undergoing total or near-total colectomy and TERPT, three factors (rapid transit time, ${ }^{[15]}$ no colonic enzyme inactivation, ${ }^{[13]}$ high enzyme concentration due to infancy ${ }^{[14]}$ ) and probably intraoperative sphincter dilatation, even careful operative technique contribute to a high stool frequency and proteolytic activity in feces, predisposing them to perianal dermatitis. ${ }^{[13-15]}$

The most important fecal proteases (trypsin, $\alpha$-chymotrypsin and elastase) can be inhibited by potato-derived protease inhibitors. ${ }^{[14]}$ In infants with Hirschsprung's disease, this therapeutic concept has not yet been evaluated. We studied this novel protease inhibitor in four children with severe perianal dermatitis (after TERPT for long segment Hirschsprung's disease) refractory to all conventional treatments.

\section{Methods}

A prospective study was approved by the ethical committee of the Children's Hospital, Inselspital, 
University of Bern. The parents of four children with perianal dermatitis after TERPT for long-segment Hirschsprung's disease gave informed consent to topical treatment with a potato-derived protease inhibitor (PPI). A trial with PPI was initiated if dermatitis persisted for more than 4 months after the pull-through. Prior to PPI administration, all four children were unsuccessfully treated for months with several ointments and systemic medication. For at least 4 weeks before participating in the study, all patients used the carrier zinc oxide ointment (Riccovitan ${ }^{\circledR}$ (patient 1) or Zincream Medinova ${ }^{\circledR}$ (patients 2-4)) without PPI. Therefore, the skin condition at the beginning of the study represents a control status of identical treatment without PPI. The only parameter that changed during the study was the addition of PPI to the previously employed zinc oxide ointment.

After exclusion of a history of hypersensitive skin reactions, an irritation test on the left upper arm was carried out two days before starting the treatment on the perianal region. Parents of the patients were asked to apply the PPI with each diaper-change and to record the times of application. They also noted daily a subjective dermatitis score ( 0 point: healthy skin, 10 points: worst dermatitis observed). Photodocumentation was made with visits to the hospital before, during and at the end of the treatment.

In all 4 patients, an ostomy was initially made because of long distance aganglionosis. The operation with TERPT and ostomy take-down was performed in a second stage. Patient 1 was a 2-year-old boy suffering from Zuelzer-Wilson syndrome (aganglionosis until terminal ileum and dysganglionosis until mid-ileum) and received a total colectomy, resection of the terminal ileum, and an ileoanal anastomosis. PPI treatment was initiated 14.5 months after TERPT. Patient 2 was a boy aged 1.5 years with the colon resected from the right colonic flexure to the rectum since aganglionosis extended into the transverse colon. PPI treatment was started 10.5 months after the pull-through. Patients 3 and 4 were twin boys of 1 year old, suffering from familial Hirschsprung's disease. In both, aganglionosis extended to the transverse colon and a TERPT with an ascendo-rectostomy was performed. PPI treatment was started 4 months after the operation.

PPI was prepared from potatoes (Bintje) as described previously ${ }^{[14]}$ resulting in a lyophilized $75 \%$ protein fraction that was tested for sterility. The potato protein-extract was added either to Riccovitan ${ }^{\circledR}$ (patient 1) or to Zincream Medinova ${ }^{\circledR}$ (patients 2-4), at a concentration of $1 \%(\mathrm{w} / \mathrm{w})$.

\section{Results}

PPI-ointment was simple to apply and no adverse effects were observed either during the hypersensitivity testing on normal skin or in the affected perianal skin areas. The PPI-ointment was stored at $4^{\circ} \mathrm{C}$ in a household refrigerator. The dermatitis scores and application frequencies for patients 1-4 are shown in Fig. 1. The pre- and post-treatment photographs of each patient showing improvement of the dermatitis in 3 out of the 4 patients (Fig. 2).

In patient 1 , an improvement was noted from score 8 to score 3 during the first 7 days. After that, the score decreased to 2 on days 15 and 16. During this time the boy was asleep for $\geq 7$ hours for 6 nights, whereas prior to the treatment, he woke his parents up to 10 times per night. At day 17, the score increased again. However, the mother reported that pain after passing stools decreased and that he slept for more than 7 hours for several nights.

In patient 2 , the dermatitis score decreased from 8 to 2 within 3 weeks. From day 24 to 27 a score of 5 was seen; thereafter, the score remained at 2 . The parents reported that the child slept better at night.
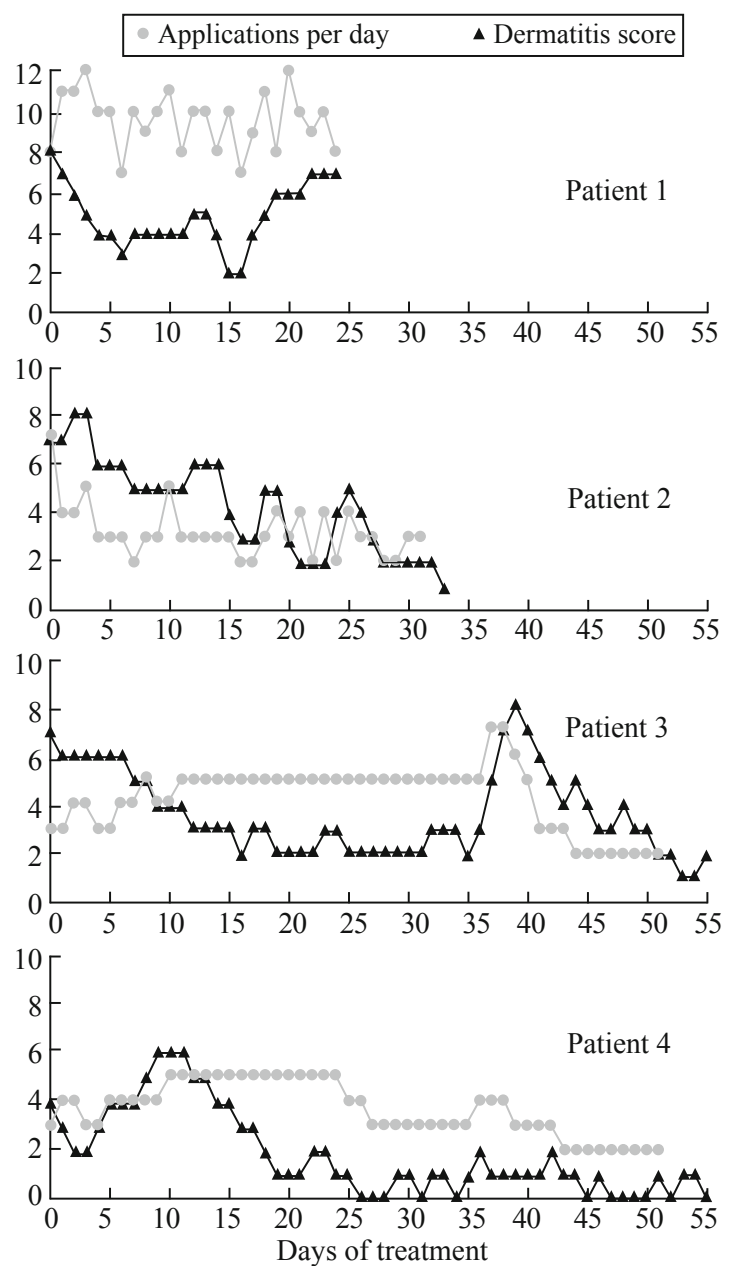

Fig. 1. Time course of daily evaluation by dermatitis score and frequency of application per day during the treatment with potato derived protease inhibitor in the four patients. 


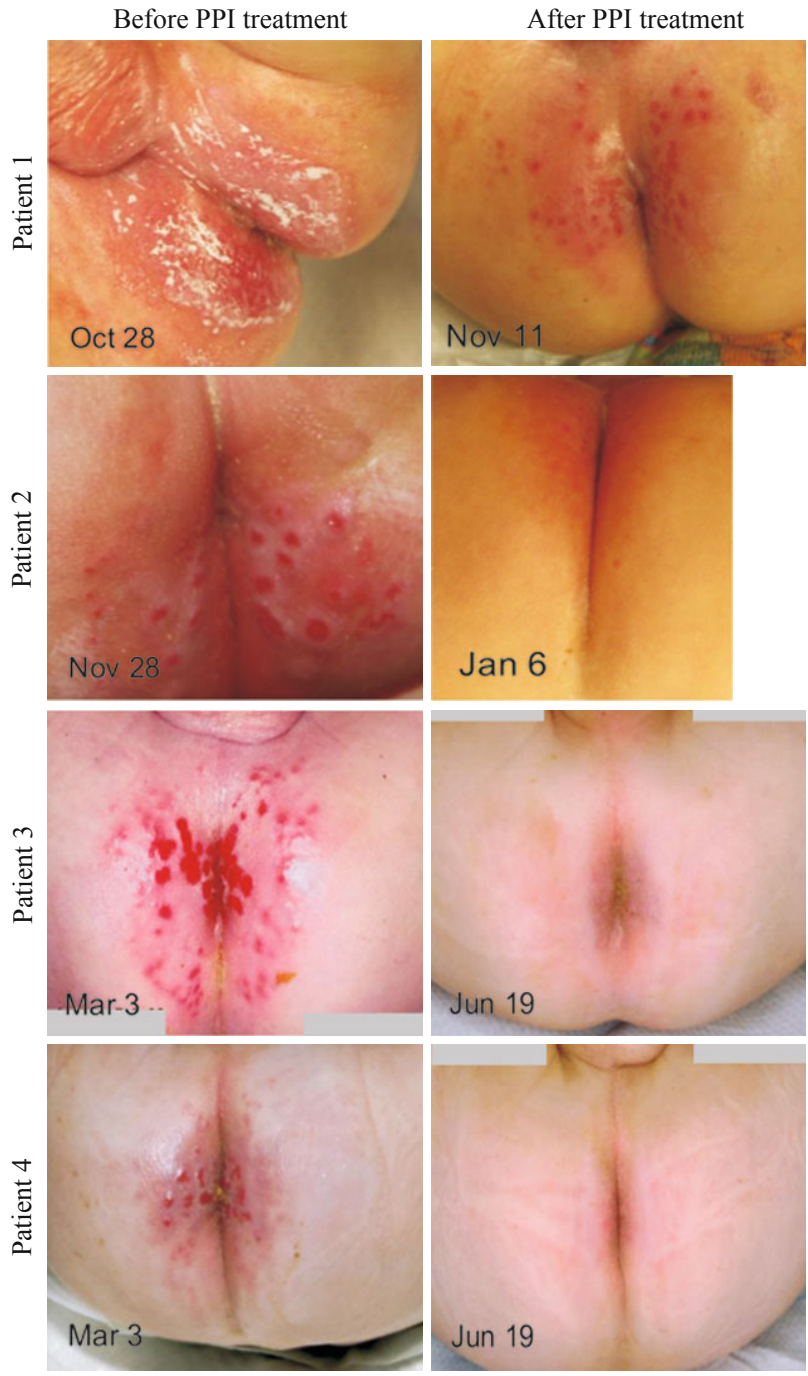

Fig. 2. Photographs of perianal skin conditions in patients 1-4 before and after 3-10 weeks of potato derived protease inhibitor treatment.

In patient 3 , the score decreased from 7 to 2 during the first 17 days. From day 17 to day 36, the score remained at 2-3. On days 37 and 38, the boy suffered from diarrhea and the score reached 8 on day 39 , then dropped again to 1-2 on days 51-54.

In patient 4 , an improvement was seen during the first 5 days (from score 4 to 2 ), but then, deterioration to score 6 occurred on day 9 , followed by an improvement to score 1 on day 19. After day 19, the score remained low.

\section{Discussion}

Perianal dermatitis is a common complication after TERPT ${ }^{[6,7]}$ particularly in infants with long-segment Hirschsprung's disease since they have several factors contributing to the development of dermatitis.

Firstly, even after resection of short segment
Hirschsprung's and TERPT, frequent bowel movements are usually observed for a period of 1-3 months. ${ }^{[8,9]}$ This effect is, however, of temporary nature since sphincter strength was recently shown to recover completely by 14 months after the operation. ${ }^{[10]}$ Secondly, the already high activity of pancreatic proteolytic enzymes in feces of healthy infants increases further after a resection of the colon. ${ }^{[14]}$ Thirdly, inside a diaper, feces and urine may have the following effects: fecal enzymes increase the permeability of the skin for bile salts, contributing to skin irritation, ${ }^{[16]}$ urea itself leads to hyperhydration of the skin, making it more permeable and irritable, and fecal urease produces ammonia from urea, which increases the $\mathrm{pH} .{ }^{[16]}$ The elevated $\mathrm{pH}$ contributes to the activation of fecal lipases, ${ }^{[15]}$ which attack the intercellular lipid lamellae of the stratum corneum. So far, no specific treatment for this unpleasant condition is available. There are numerous unspecific treatments such as zinc or titanium oxide creme, steroid or antimycotic ointments, frequent diaper changes and other conventional measures such as painting with gentian violet, bathing, or drying the skin with a hairdryer. Pharmacotherapy has been tried with oral antibiotics, antimycotics, and antiperistaltic drugs. However, none of these treatments, either alone or in combination, was effective.

The protease inhibitor selected for the current study is unique in its ability to inhibit all pancreatic proteases that are clinically important in this context. ${ }^{[14]}$ However, its isolation is a time-consuming process, limiting the number of patients that can be treated in a pilot study. Adverse effects were neither seen during the hypersensitivity testing on normal skin nor in the affected perianal skin areas, and parents appreciated the use of a "natural" potato protein preparation.

A remarkable improvement in the skin condition of 3 of the 4 children with long-segment Hirschsprung's disease after colon resection and TERPT was observed after administration of the PPI ointment for several weeks. In two patients (patients 3 and 4), this effect lasted beyond the application period. Besides the effects on dermatitis score, an improvement of sleep disturbance in patients 1 and 2 was observed. This effect was not quantified, except by the reduced frequency of PPI-applications documented at night. The reduced pain after defecation of the children was a source of relief for the parents.

To our knowledge this is the first clinical study on the topical application of PPI with a focus on the possible therapeutic effects. There are also other possible applications of PPI, such as common diaper rash in infants, dermatitis after colectomy for Crohn's disease or ulcerative colitis, and patients with intestinal resection and ileostomy. We conclude that a larger trial 
of PPI in perianal dermatitis is justified because of the potential beneficial effect and a low risk of adverse effects.

\section{Acknowledgements}

We thank Dr. Nicolas Martin Shaw and Prof. Dr. Bob Friis for their careful revision of the manuscript. We thank the Institute of Hospital Pharmacy (Head Dr. Jeanette Goette) of Inselspital University of Berne for preparation of the ointments used in this trial.

\section{Funding: None.}

Ethical approval: The study was approved by the ethical committee of the Children's Hospital, Inselspital, University of Berne.

Competing interest: No conflicts of interest to be declared.

Contributors: Berger S developed the concept and designed the study. Berger S and Rufener A wrote the first draft, Klimek P and Zachariou $\mathrm{Z}$ analyzed the data and revised the paper. Boillat $\mathrm{C}$ produced the protease inhibitor, interpreted the data, and revised the paper.

\section{References}

1 Escobar MA, Grosfeld JL, West KW, Scherer LR, Rouse TM, Engum SA, et al. Long-term outcomes in total colonic aganglionosis: a 32-year experience. J Pediatr Surg 2005;40:955961.

2 Wildhaber BE, Teitelbaum DH, Coran AG. Total colonic Hirschsprung's disease: a 28-year experience. J Pediatr Surg 2005;40:203-206.

3 Rodriguez Cano L, Garcia-Patos Briones V, Pedragosa Jove R, Castells Rodellas A. Perianal pseudoverrucous papules and nodules after surgery for Hirschsprung disease. J Pediatr 1994;125:914-916.

4 Rodriguez-Poblador J, Gonzalez-Castro U, Herranz-Martinez S, Luelmo-Aguilar J. Jacquet erosive diaper dermatitis after surgery for Hirschsprung disease. Pediatr Dermatol 1998;15:46-47.

5 Bourrat E, Vaquin C, Prigent F, Rybojad M. Perianal papulonodular dermatitis in Hirschsprung disease. Ann Dermatol Venereol 1996;123:549-551.

6 Langer JC, Seifert M, Minkes RK. One-stage Soave pull-through for Hirschsprung's disease: a comparison of the transanal and open approaches. J Pediatr Surg 2000;35:820-822.

7 Langer JC, Durrant AC, de la Torre L, Teitelbaum DH, Minkes RK, Caty MG, et al. One-stage transanal Soave pullthrough for Hirschsprung disease: a multicenter experience with 141 children. Ann Surg 2003;238:569-583.

8 Teitelbaum D, Coran A. Primary pull-through in the newborn. Seminars Pediatr Surg 1998;7:103-107.

9 Zhang SC, Bai YZ, Wang W, Wang WL. Clinical outcome in children after transanal 1-stage endorectal pull-through operation for Hirschsprung disease. J Pediatr Surg 2005;40:1307-1311.

10 Till H, Heinrich M, Schuster T, V Schweinitz D. Is the anorectal sphincter damaged during a transanal endorectal pull-through (TERPT) for Hirschsprung's disease? A 3-dimensional, vector manometric investigation. Eur J Pediatr Surg 2006;16:188-191.

11 Layer P, Keller J. Pancreatic enzymes: secretion and luminal nutrient digestion in health and disease. J Clin Gastroenterol 1999;28:3-10.

12 Layer P, Go V, DiMagno E. Fate of pancreatic enzymes during small intestinal aboral transit in humans. Am J Physiol 1986;251:475-480.

13 Gibson S, McFarlan C, Hay S, MacFarlane G. Significance of microflora in proteolysis in the colon. App Environ Microbiol 1989;55:679-683.

14 Ruseler-van Embden JG, van Lieshout LM, Smits SA, van Kessel I, Laman JD. Potato tuber proteins efficiently inhibit human faecal proteolytic activity: implications for treatment of peri-anal dermatitis. Eur J Clin Invest 2004;34:303-311.

15 Atherton DJ. A review of the pathophysiology, prevention and treatment of irritant diaper dermatitis. Curr Med Res Opin 2004;20:645-649.

16 Halbert AR, Chan JJ. Anogenital and buttock ulceration in infancy. Australas J Dermatol 2002;43:1-6.

Received December 8, 2010 Accepted after revision May 11, 2011 